\title{
Adherence to Antiretroviral Therapy and Associated Factors among HIV Infected Patients in Nigist Eleni Mohammed Memorial General Hospital, Hossana, Southern Ethiopia
}

\author{
Bruke Berhanu Billoro ${ }^{1 *}$, Girma Mamo² and Habtemu Jarso² \\ ${ }^{1}$ Department of Pharmacy, College of medicine and health sciences, Wachemo University, Hosanna, Ethiopia \\ ${ }^{2}$ School of Pharmacy, College of Health Sciences, Jimma University, Jimma, Ethiopia
}

\begin{abstract}
Background: Adherence to antiretroviral therapy is a major predictor of the success of HIVIAIDS treatment. Good adherence to antiretroviral therapy is necessary to achieve the best virology response, lower the risk of drug resistance and reduce morbidity and mortality.
\end{abstract}

Objective: To determine adherence rate to antiretroviral therapy and identify associated factors among HIV infected patients accessing ART at Nigist Eleni Mohammed Memorial General Hospital, Hosanna; Southern Ethiopia.

Method: We performed a cross-sectional study involving two hundred sixty-five adult patients on anti-retroviral therapy at Nigist Eleni Mohammed Memorial General Hospital from March to April 2015. Data were extracted by face to face exit-interview of the patient and reviewing they are respective follow-up record using structured questionnaire and checklist respectively Data were analyzed using Statistical Package for Social Science (SPSS) for window version 20.0 software. Predictors of adherence were identified using multivariable logistic regression model. A p-value less than 0.05 were considered to be statistically significant. Finally, the results were presented by text, tables, and chart.

Results: We studied 265 patients and response rate was 99.6\%. $149(56 \%)$ respondents were adhered in the week prior to interview. Disclosing ART use [AOR=2.48 $(1.28,4.79)]$, no substance abuse $[A O R=8.01(2.15,29.84)]$, being married $[A O R=2.35(1.10,5.04)]$, medication schedule fit daily activity $[A O R=5.05(1.79,14.21)]$, following specific medication schedule [AOR=2.41 $(1.11,5.24)]$, absence of side-effects [AOR=3.89 $(2.11,7.19)$, having access to health center $[A O R=2.13(1.18,3.86)]$ were independent predictor of adherence. Forgetfulness and being busy were the most common reasons for missing doses.

Conclusion: The ART adherence level was relatively low. Appropriate adherence-enhancing strategies targeted at creating awareness on the side effect, substance use, disclosure status and medication fitness with daily activity is strongly recommended.

Keywords: HAART; Adherence; Predictors; Substance use; Ethiopia

\section{Introduction}

Human Immunodeficiency Virus, the pathogen that causes Acquired Immune Deficiency Syndrome has been the most significant emerging infectious agent in the last century and continually threatens to create health, social and developmental problems in this millennium [1]. In 2015 there were 2.1 million ( 1.8 million-2.4 million) new HIV infections worldwide, adding up to a total of 36.7 million $(34.0$ million-39.8 million) people living with HIV [2].

Medical knowledge around HIV/AIDS has increased significantly over the years and good progress has been made in the treatment of HIV as a manageable life-threatening chronic condition using antiretroviral therapy [3]. ART has also remained the only available option that offers the possibility of dramatically reducing HIV/AIDS-related morbidity and mortality [4].

Adherence is defined as the patient's ability to follow treatment plan by taking the correct dose of medications, at prescribed time and frequencies (on schedule) and following dietary instructions [5]. Adherence to HAART is one of the potentially alterable factors determining outcomes for patients with HIV [6]. However, non-adherence to HAART is common in all groups of treated individuals [7]. To achieve goal of HAART, maximal and durable viral suppression and successful HIV therapy requires adherence of $\geq 95 \%[8]$.
Good adherence is crucial for maximum clinical benefit from ART. However, studies are rare in Ethiopia regarding prevalence and predictors of adherence to ART. There is no study done in Nigist Eleni Mohammed Memorial General Hospital (NEMMGH) regarding prevalence and predictors of adherence to ART. Therefore, this study aimed to show the adherence rate and predictors of adherence to ART in ART clinic of NEMMGH. The result of the our study will provide baseline information for health professionals, governmental and non-governmental organizations working in health care system of NEMMGH as well as in the country to decrease non-adherence, with the ultimate goal of improving the effectiveness of HIV treatment through adherence at Southern Ethiopia and in the meantime complications related with non-adherence.

*Corresponding author: Bruke Berhanu Billoro, Department of Pharmacy, College of medicine and health sciences, Wachemo University, Hosanna, Ethiopia; Tel: +251913231628/+251954746688; E-mail: 3b2005pharm@gmail.com

Received June 18, 2018; Accepted August 23, 2018; Published August 30, 2018

Citation: Billoro BB, Mamo G, Jarso H (2018) Adherence to Antiretroviral Therapy and Associated Factors among HIV Infected Patients in Nigist Eleni Mohammed Memorial General Hospital, Hossana, Southern Ethiopia. J AIDS Clin Res 9: 774 doi: 10.4172/2155-6113.1000774

Copyright: $\odot 2018$ Billoro BB, et al. This is an open-access article distributed under the terms of the Creative Commons Attribution License, which permits unrestricted use, distribution, and reproduction in any medium, provided the original author and source are credited. 


\section{Methods and Materials}

\section{Study design and participants}

Institution based cross-sectional study was conducted from March 27, 2015, to April 27, 2015. Source population was all adult PLWHA who were on ART follow up at NEMMGH, which is one of the hospitals and serve as a referral center for in Hadiya zone.

The study population was all adult patients on ART who visits ART pharmacy during the study period and fulfill the eligibility criteria of the study subject. All adult patients who have followed up for at least 2 month, Age $>15$ years and Patients giving consent were included. Those who had incomplete records, missing clinical records and those with hearing or speaking problem, mentally unstable were excluded. The sample size was determined using a single population proportion formula; considering 5\% marginal error, $95 \%$ level of confidence and using $74.4 \%$ proportion of adherence in study done in Wolaita Sodo hospital [9]. HIV/AIDS patients on ART at NEMMGH were 1405, correcting for finite population and considering $10 \%$ for possible none response rate. The final sample size was $265 \mathrm{HIV} / \mathrm{AIDS}$ patients on ART were included in the study. Participants were selected by consecutive sampling.

Adherence to ART: was when the patients took $\geq 95 \%$ of the prescribed doses in a week prior to the study.

Non-adherence: was when the patients took $<95 \%$ of the prescribed doses in a week prior to the study.

Adherence rate: the number of doses taken (the number of doses prescribed minus number of dose missed) divided by the number of doses prescribed multiplied by 100 .

Data collection instrument were adopted from previous similar studies [9-12].

Data was collected by face to face exit-interview of the patients and reviewing they are respective follow-up records using structured questionnaire and checklist respectively. The checklist contains information about some of the medication-related factors and diseaserelated factors.

Data were collected by two trained Nurses (BSc) not working at ART clinic using pretested data collection tool prepared by the research team. The research team supervised the data collection process daily. Socio-demographic and other patient-related factors were obtained directly from patients and patient's medical chart.

The variables included a dependent variable which is adherence status to ART, and independent variables are socio-demographic characteristics, patient-related variables, medication-related variables, health professionals and clinical setting related variables and diseaserelated variables.

\section{Statistical methods}

Data were coded and cleaned using Epi-Data version 3.1 and exported to SPSS for window version 20.0 for analysis. Descriptive statistics were used to present socio-demographic, Patient-related factors, Medication-related factors. We used percentages, frequencies, means, standard deviation and cross tabulation to describe patient characteristics, medication-related factors and to determine the relationship between dependent and independent variables. In addition, bivariate and multivariable backward logistic regression with unadjusted and adjusted odds ratio was used to identify factors associated with adherence. Before running a multivariable binary logistic regression, multicollinearity between the independent variables was checked in linear regression by Variance Inflation Factors (VIF). Variables which have a significant association at $\mathrm{p}$-value $<0.25$ in the bivariate logistic regression model were fitted to multivariate logistic regression model to identify predictors of adherence. The finding was presented using unadjusted and adjusted odds ratio and their 95\% CI. $\mathrm{P}$-value of less than 0.05 was considered as statistically significant in the multivariable analysis.

\section{Ethics approval}

Ethical clearance was obtained from Jimma University, College of Health Sciences Ethical review board. We obtained permission from Hospital management before starting data collection. Written informed consent was obtained from each study participant before data collection. The Consent was documented on the prepared format. Before starting the study, the protocol including the written consent was approved by an Ethical review board of Jimma University. We kept the information confidential.

\section{Results}

\section{Sociodemographic related factors}

Two hundred sixty-five patients full filled the inclusion criteria and the analysis was done on a total of 265 patients that fulfilled the inclusion and exclusion criteria.

The majorities $(62.6 \%)$ were females and the mean age of the patients was $34 \pm 9.6$. More than half of patients (55.8\%) were married (Table 1).

\begin{tabular}{|c|c|c|c|}
\hline \multicolumn{2}{|c|}{ Socio-demographic characteristics } & \multirow{2}{*}{$\begin{array}{c}\text { Frequency } \\
122\end{array}$} & \multirow{2}{*}{$\begin{array}{c}\text { Percentage (\%) } \\
46\end{array}$} \\
\hline Age (years) & $15-30$ & & \\
\hline & $31-45$ & 122 & 46 \\
\hline & $\geq 46$ & 21 & 8 \\
\hline \multirow[t]{2}{*}{ Sex } & Male & 99 & 37.4 \\
\hline & Female & 166 & 62.6 \\
\hline \multirow[t]{4}{*}{ Ethnicity } & Amhara & 45 & 17.0 \\
\hline & Gurage & 23 & 8.7 \\
\hline & Hadiya & 147 & 55.5 \\
\hline & Others & 50 & 18.9 \\
\hline \multirow[t]{2}{*}{ Residence } & Urban & 157 & 59.2 \\
\hline & Rural & 108 & 40.8 \\
\hline \multirow[t]{4}{*}{ Religion } & Orthodox & 89 & 33.6 \\
\hline & Protestant & 155 & 58.5 \\
\hline & Muslim & 15 & 5.7 \\
\hline & Others & 6 & 2.3 \\
\hline \multirow[t]{4}{*}{ Educational level } & No formal education & 30 & 11.3 \\
\hline & $1-8$ & 143 & 54.0 \\
\hline & $9-12$ & 71 & 26.8 \\
\hline & College and above & 21 & 7.9 \\
\hline \multirow{4}{*}{ Marital status } & Single & 40 & 15.1 \\
\hline & Married & 148 & 55.8 \\
\hline & Divorced & 27 & 10.2 \\
\hline & Windowed & 50 & 18.9 \\
\hline \multirow[t]{2}{*}{ Living condition } & With family or friends & 211 & 79.6 \\
\hline & Alone & 54 & 20.4 \\
\hline \multirow[t]{2}{*}{ Occupational status } & Employed & 29 & 10.9 \\
\hline & Unemployed & 236 & 89.1 \\
\hline
\end{tabular}

Table 1: Socio-demographic characteristics of the respondents on HAART in NEMMGH, ART Clinic, South Ethiopia ( $n=265)$, March 27 to April 27, 2015. 


\section{Patient-related factors}

One hundred ninety-one $(72.1 \%)$ had disclosure status of using ART to their family members or friends.one hundred eighty seven (70.6\%) participants were helped by their family members or friends in remembering to take their medications. 238 (89.8\%) participants did not use memory aid and $241(90.9 \%)$ participants had no exposure to the substance (Table 2).

The main reason for not following clinical appointment regularly was; $41(35.7 \%)$ transportation problem and $34(29.6 \%)$ side effect respectively (Figure 1).

\begin{tabular}{|c|c|c|c|}
\hline \multicolumn{2}{|l|}{ Patient related variables } & \multirow{2}{*}{\begin{tabular}{|c|} 
Frequency \\
191 \\
\end{tabular}} & \multirow{2}{*}{$\begin{array}{c}\text { Percentage (\%) } \\
72.1\end{array}$} \\
\hline Disclosure status & Yes & & \\
\hline & No & 74 & 27.9 \\
\hline Family or friend support & $\begin{array}{l}\text { Yes } \\
\text { No }\end{array}$ & $\begin{array}{c}187 \\
78\end{array}$ & $\begin{array}{l}70.6 \\
29.4\end{array}$ \\
\hline \multirow[t]{3}{*}{ Frequency of Family or friend support } & All times & 53 & 20.0 \\
\hline & Some times & 134 & 50.6 \\
\hline & Never & 78 & 29.4 \\
\hline \multirow{2}{*}{$\begin{array}{l}\text { Frequency of following specific } \\
\text { medication time schedule }\end{array}$} & All times & 216 & 81.5 \\
\hline & Some times & 49 & 18.5 \\
\hline \multirow{2}{*}{$\begin{array}{l}\text { Follow instruction given by health } \\
\text { professional }\end{array}$} & Yes & 264 & 99.6 \\
\hline & No & 1 & 0.4 \\
\hline \multirow[t]{2}{*}{ Medication schedule fit daily activity } & Yes & 238 & 89.8 \\
\hline & No & 27 & 10.2 \\
\hline \multirow[t]{2}{*}{ Sharing the medications } & Yes & 1 & 0.4 \\
\hline & No & 264 & 99.6 \\
\hline \multirow{2}{*}{$\begin{array}{l}\text { Longer duration of therapy has } \\
\text { negative impact on treatment }\end{array}$} & Yes & 255 & 96.2 \\
\hline & No & 10 & 3.8 \\
\hline \multirow[t]{2}{*}{ Regular follow up } & Yes & 150 & 56.6 \\
\hline & No & 115 & 43.4 \\
\hline \multirow[t]{2}{*}{ History of substance abuse } & Yes & 24 & 9.1 \\
\hline & No & 241 & 90.9 \\
\hline \multirow[t]{2}{*}{ Food restriction } & Yes & 113 & 42.6 \\
\hline & No & 152 & 57.4 \\
\hline \multirow[t]{2}{*}{ Use of reminding aid } & Yes & 27 & 10.2 \\
\hline & No & 238 & 89.8 \\
\hline
\end{tabular}

Table 2: Patient related variables affecting participants' adherence in NEMMGH, ART Clinic, South Ethiopia ( $n=265$ ), March 27 to April 27, 2015

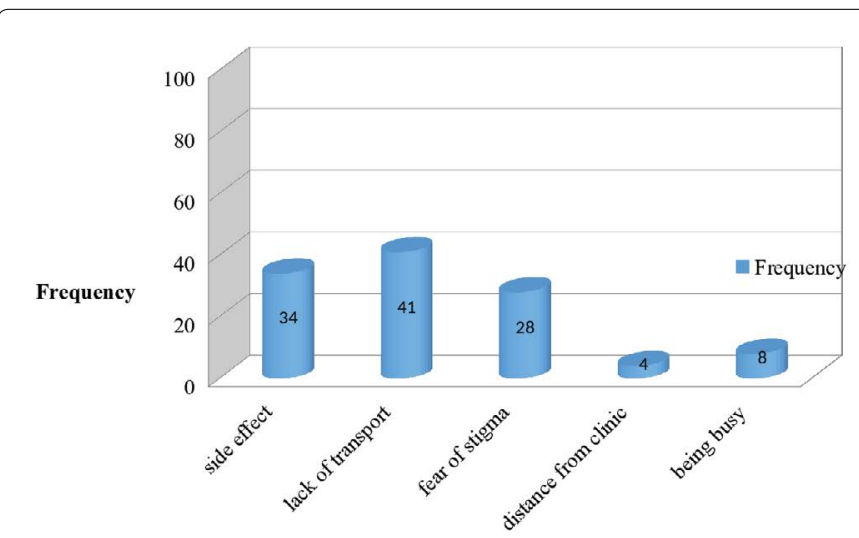

Figure 1: Reasons given for not following clinical appointment regularly among respondents at NEMMGH, ART clinic, South Ethiopia $(n=265)$ March 27 to April 27, 2015.

\section{Medication-related factors}

Two hundred thirty-eight (89.8\%) of participants took anti-retroviral drugs for more than 1 years and 4 (1.5\%) of participants took the HAART less than 3 months. $111(41.9 \%)$ of the participants were started on $\mathrm{TDF} / 3 \mathrm{TC} / \mathrm{EFV}$ regimen during the initiation of ART and 127 (47.9\%) of the participants were currently on TDF/3TC/EFV regimen (Table 3).

\section{Health professionals and clinical setting related factors}

Two hundred sixty-four (99.9\%) of the participants believed that interaction with caregivers improves the outcome of treatment. Of all study participants, $262(95.6 \%)$ participants privacy was maintained during treatment. Regarding health professionals' patient's relationship, $227(85.7 \%)$ of the study participants had a good relationship with their health professionals (Table 4).

\begin{tabular}{|c|c|c|c|}
\hline \multicolumn{2}{|l|}{ Medication-related variables } & \multirow{2}{*}{$\begin{array}{c}\text { Frequency } \\
4\end{array}$} & \multirow{2}{*}{$\begin{array}{c}\text { Percentage }(\%) \\
1.5\end{array}$} \\
\hline Duration of ART & $<3$ months & & \\
\hline & $3-6$ months & 9 & 3.4 \\
\hline & $6-12$ months & 14 & 5.3 \\
\hline & $>12$ months & 238 & 89.8 \\
\hline \multirow[t]{2}{*}{ Other medication } & Yes & 201 & 75.8 \\
\hline & No & 64 & 24.2 \\
\hline $\begin{array}{l}\text { Type of other medication } \\
\text { used }\end{array}$ & $\begin{array}{c}\text { Anti-TB } \\
\text { Cotrimoxazole } \\
\text { Fluconazole } \\
\text { Others }\end{array}$ & $\begin{array}{c}46 \\
111 \\
15 \\
29\end{array}$ & $\begin{array}{c}22.9 \\
55.2 \\
7.5 \\
14.4\end{array}$ \\
\hline \multirow[t]{2}{*}{ Side effect faced } & Yes & 135 & 50.9 \\
\hline & No & 130 & 49.1 \\
\hline \multirow[t]{7}{*}{ Initial ART Regimen } & D4T/3TC/NVP & 48 & 18.1 \\
\hline & ZDV/3TC/NVP & 43 & 16.2 \\
\hline & TDF/3TC/EFV & 111 & 41.9 \\
\hline & D4T/3TC/EFV & 16 & 6.0 \\
\hline & TDF/3TC/NVP & 19 & 7.2 \\
\hline & ZDV/3TC/EFV & 25 & 9.4 \\
\hline & Others & 3 & 1.1 \\
\hline \multirow{5}{*}{ Current } & ZDV/3TC/NVP & 75 & 28.3 \\
\hline & TDF/3TC/EFV & 127 & 47.9 \\
\hline & TDF/3TC/NVP & 18 & 6.8 \\
\hline & ZDV/3TC/EFV & 36 & 13.6 \\
\hline & Others & 9 & 3.4 \\
\hline
\end{tabular}

Table 3: Medication-related variables affecting the study participants' adherence to HAART in NEMMGH, ART Clinic, South Ethiopia ( $n=265$ ), March 27 to April 27 , 2015.

\begin{tabular}{|c|c|c|c|}
\hline \multicolumn{2}{|l|}{$\begin{array}{l}\text { Health professional and clinical setting } \\
\text { related variables }\end{array}$} & \multirow{2}{*}{$\begin{array}{c}\text { Frequency } \\
265\end{array}$} & \multirow{2}{*}{$\begin{array}{c}\text { Percentage (\%) } \\
100\end{array}$} \\
\hline Told about the problems of low adherence & Yes & & \\
\hline $\begin{array}{l}\text { Communication with caregiver improves } \\
\text { the outcome of treatment }\end{array}$ & $\begin{array}{l}\text { Yes } \\
\text { No }\end{array}$ & $\begin{array}{c}264 \\
1\end{array}$ & $\begin{array}{c}99.9 \\
0.4\end{array}$ \\
\hline Counseling during treatment & $\begin{array}{l}\text { Yes } \\
\text { No }\end{array}$ & $\begin{array}{c}264 \\
1\end{array}$ & $\begin{array}{c}99.9 \\
0.4\end{array}$ \\
\hline Privacy maintained during treatment & $\begin{array}{l}\text { Yes } \\
\text { No }\end{array}$ & $\begin{array}{c}262 \\
3\end{array}$ & $\begin{array}{c}98.9 \\
1.1\end{array}$ \\
\hline $\begin{array}{l}\text { Good health professional-patient } \\
\text { relationship }\end{array}$ & $\begin{array}{l}\text { Yes } \\
\text { No }\end{array}$ & $\begin{array}{c}227 \\
38\end{array}$ & $\begin{array}{l}85.7 \\
14.3\end{array}$ \\
\hline Having access to the medical center & $\begin{array}{l}\text { Yes } \\
\text { No }\end{array}$ & $\begin{array}{l}144 \\
121\end{array}$ & $\begin{array}{l}54.3 \\
45.7\end{array}$ \\
\hline
\end{tabular}

Table 4: Health professional and clinical setting related variables affecting adherence of study participants to HAART in NEMMGH, ART Clinic, South Ethiopia $(n=265)$, March 27 to April 27, 2015. 


\section{Disease-related factors}

Two hundred twenty-six (85.3\%) patients had a current CD4 count greater than 200 cells $/ \mathrm{mm}^{3}$. Out of 258 study subjects, $92(35.7 \%)$ of the study subjects were in WHO stage I during initiation while $29(11.2 \%)$ were on advanced stage (stage IV). $7(2.6 \%)$ and $2(0.8 \%)$ of clients have no recorded WHO stage during initiation and recently respectively (Table 5).

\section{Adherence rate}

One hundred forty-nine (56.2\%) patients had taken prescribed ART a week before the study period and 116 (43.8\%) patients had missed some of the prescribed pills a week before the study period

Then adherence rate of the patients a week before the study period was calculated by the formula:

Adherence rate $=($ Number of doses supposed to be taken-Number of doses missed)/Number of doses supposed to be taken $\mathrm{x} 100$

In this study, adherence was measured using self-report and the adherence to antiretroviral therapy was $56.2 \%$ of all prescribed doses.

Missed doses were assessed for "the day before", "three days recall" and "seven days recall". $22.3 \%$ of clients missed at least a single dose of drug the day before, $31.3 \%$ missed at least a dose in three days recall, while 32.8 missed at least a dose in seven days recall (Table 6).

\begin{tabular}{|c|c|c|c|}
\hline \multicolumn{2}{|l|}{ Disease-related variable } & Frequency & Percentage (\%) \\
\hline Improvement since initiation of ART & Yes & 265 & 100 \\
\hline A tendency to continue to take medication & $\begin{array}{l}\text { Yes } \\
\text { No }\end{array}$ & $\begin{array}{c}264 \\
1\end{array}$ & $\begin{array}{c}99.6 \\
0.4\end{array}$ \\
\hline \multirow[t]{2}{*}{ CD4 cell count } & $<200$ & 64 & 34.2 \\
\hline & $\geq 200$ & 123 & 65.8 \\
\hline \multirow[t]{2}{*}{ current } & $<200$ & 39 & 14.7 \\
\hline & $\geq 200$ & 226 & 85.3 \\
\hline \multirow[t]{4}{*}{ WHO clinical stage } & stage I & 92 & 35.7 \\
\hline & $\begin{array}{c}\text { stage } \\
\text { II }\end{array}$ & 85 & 32.9 \\
\hline & $\begin{array}{l}\text { stage } \\
\text { III }\end{array}$ & 52 & 20.2 \\
\hline & $\begin{array}{l}\text { stage } \\
\text { IV }\end{array}$ & 29 & 11.2 \\
\hline \multirow{4}{*}{ current } & stage I & 158 & 60.1 \\
\hline & $\begin{array}{c}\text { stage } \\
\text { II }\end{array}$ & 79 & 30.1 \\
\hline & $\begin{array}{l}\text { stage } \\
\text { III }\end{array}$ & 23 & 8.7 \\
\hline & $\begin{array}{l}\text { stage } \\
\text { IV }\end{array}$ & 3 & 1.1 \\
\hline
\end{tabular}

Table 5: Disease-related variables of the study participants on ART in NEMMGH, ART Clinic, South Ethiopia ( $n=265$ ), March 27 to April 27, 2015.

\begin{tabular}{|l|c|c|c|}
\hline \multicolumn{2}{|l|}{ Number of days } & Frequency & Percentage (\%) \\
\hline Previous day & Yes & 59 & 22.3 \\
\cline { 2 - 4 } & No & 206 & 77.7 \\
\hline \multirow{2}{*}{ Past three days } & Yes & 83 & 31.3 \\
\cline { 2 - 4 } & No & 182 & 68.7 \\
\hline Past seven days & Yes & 87 & 32.8 \\
& No & 178 & 67.2 \\
\hline Self-reported 7 days recall dose adherence & $\geq 95 \%$ & 149 & 56.2 \\
rate & $<95 \%$ & 116 & 43.8 \\
\hline
\end{tabular}

Table 6: Distribution of adherence rate and Patients who missed a dose by selfreport in NEMMGH, ART clinic, South Ethiopia ( $n=265)$, March 27 to April 27, 2015.

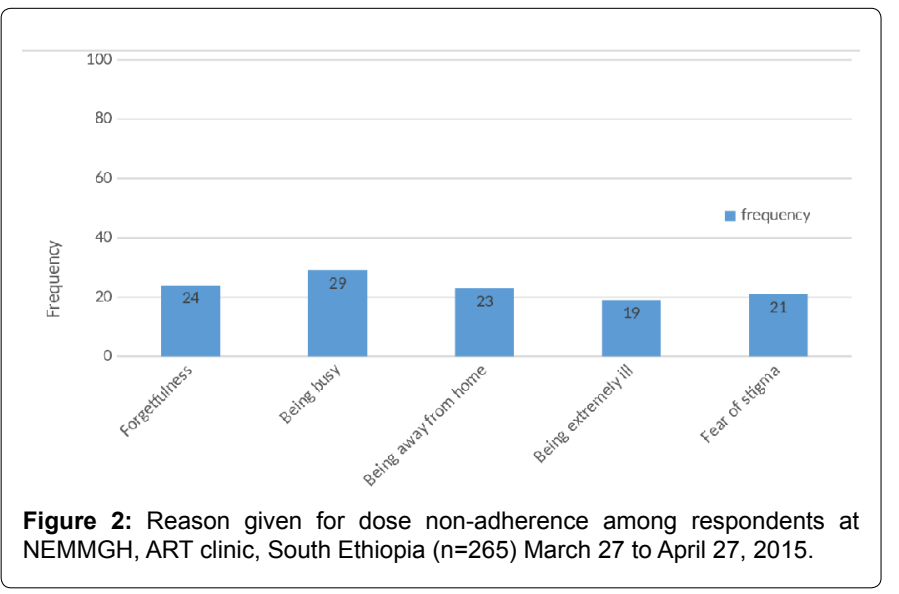

Being busy 29 (25\%) and forgetting 24 (20.7\%) are the major reasons for dose non-adherence. Also, the participants reported that being away from home $23(19.8 \%)$ and fear of stigma $21(18.1 \%)$ as a reason for missing their dose (Figure 2).

\section{Predictors of adherence}

On bivariate logistic regression model, eleven variables were associated with adherence at $\mathrm{p}<0.25$. marital status $(\mathrm{p}=0.057)$, concomitantly used medication $(\mathrm{p}=0.022)$, drug side effect $(\mathrm{p}=0.001)$, disclosure status $(\mathrm{p}=0.018)$, substance use $(\mathrm{p}=0.001)$, family and social support $(\mathrm{p}=0.008)$ specific medication schedule, $(\mathrm{p}=0.003)$ medication schedule fit daily activity $(\mathrm{p}=0.001)$, WHO stage $(\mathrm{p}=0.032)$, access to medical center $(\mathrm{p}=0.001)$ and patient to health professional relationships $(p=0.027)$ were associated with adherence status (Table 7$)$.

Based on multicollinearity test support and disclosure were correlated. Since the most relevant variable in clinical practice was chosen to enter into the multivariate model and we choose disclosure.

Marital status, drug side effect, disclosure status, substance use, specific medication schedule, access to the medical center and medication schedule fit daily activity were independent predictors of adherence in multivariate logistic regression analysis (Table 7).

Study participants who did not face side effect were 3.89 more likely adhere to regimens as compared with those participants who faced side effects [AOR $(95 \% \mathrm{CI})=3.89(2.11-7.19)]$.

A participant who married was likely increase adherence by odds of 2.35 times as compared with those participants who were windowed [AOR (95\% CI)=2.35 (1.10-5.04)] (Table 7).

Participants who disclosed their ART use were 2.48 more likely adhere to regimens as compared with those participants who were not disclosed [AOR $(95 \% \mathrm{CI})=2.48(1.28-4.79)$ ]. Participants whose daily treatment schedule fit with daily routine activities were 5.05 more likely adhere to regimens as compared with those participants whose daily treatment schedule was not fit with daily routine activities [AOR $(95 \%$ CI) $=5.05$ (1.79- 14.21)] (Table 7).

Participants who closely follow specific medication schedule were 2.41 more likely adhere to regimens as compared with those participants who did not follow specific medication schedule [AOR $(95 \% \mathrm{CI})=2.41$ (1.11-5.24)] (Table 7).

Participants who didn't use substance use were 8.01 more likely adhere to regimens as compared with their counterparts [AOR (95\% $\mathrm{CI})=8.01(2.15-29.84]$. 


\begin{tabular}{|c|c|c|c|c|c|c|}
\hline \multirow{2}{*}{ Variables } & \multicolumn{2}{|c|}{ HAART adherence } & \multirow{2}{*}{$\begin{array}{c}\text { COR } \\
{[95 \% \mathrm{Cl}]}\end{array}$} & \multirow{2}{*}{$\begin{array}{c}\mathrm{P}- \\
\text { value }\end{array}$} & \multirow{2}{*}{$\begin{array}{c}\mathrm{AOR} \\
{[95 \% \mathrm{Cl}]}\end{array}$} & \multirow{2}{*}{$\begin{array}{c}\mathrm{P}- \\
\text { value }\end{array}$} \\
\hline & $\begin{array}{c}\text { Non-adherence } \\
\text { Frequency }(\%)(n=116)\end{array}$ & $\begin{array}{c}\text { Adherence } \\
\text { Frequency }(\%) \\
(n=149)\end{array}$ & & & & \\
\hline $\begin{array}{l}\text { Marital status } \\
\text { single } \\
\text { married } \\
\text { divorced } \\
\text { windowed }\end{array}$ & $\begin{array}{c}19(47.5) \\
57(38.5) \\
13(48.1) \\
27(54)\end{array}$ & $\begin{array}{l}21(52.5) \\
91(61.5) \\
14(51.9) \\
23(46)\end{array}$ & $\begin{array}{c}1.29(0.56-2.99) \\
1.87(0.98-3.58) \\
1.26((0.49-3.23) \\
1\end{array}$ & $\begin{array}{l}0.540 \\
0.057 \\
0.624\end{array}$ & $\begin{array}{l}1.09(0.42-2.81) \\
2.35(1.10-5.04) \\
1.33(0.46-3.85)\end{array}$ & $\begin{array}{l}0.850 \\
0.027^{*} \\
0.599\end{array}$ \\
\hline $\begin{array}{l}\text { Disclosure status } \\
\text { Yes } \\
\text { No }\end{array}$ & $\begin{array}{l}75(39.3) \\
41(55.4)\end{array}$ & $\begin{array}{l}116(60.7) \\
33(44.6)\end{array}$ & $\begin{array}{c}1.92(1.12-3.31) \\
1\end{array}$ & 0.018 & $\begin{array}{c}2.48(1.28-4.79) \\
1\end{array}$ & $0.007^{\star}$ \\
\hline $\begin{array}{l}\text { Family and social support } \\
\text { Yes } \\
\text { No }\end{array}$ & $\begin{array}{l}72(38.5) \\
44(56.4)\end{array}$ & $\begin{array}{c}115(61.5) \\
34(43.6)\end{array}$ & $\begin{array}{c}2.07(1.21-3.53) \\
1\end{array}$ & 0.008 & & \\
\hline $\begin{array}{l}\text { Following specific medication } \\
\text { time schedule } \\
\text { All time } \\
\text { Sometime }\end{array}$ & $\begin{array}{l}85(39.4) \\
31(63.3)\end{array}$ & $\begin{array}{c}131(60.6) \\
18(36.7)\end{array}$ & $\begin{array}{c}2.65(1.39-5.04) \\
1\end{array}$ & 0.003 & $\begin{array}{c}2.41(1.11-5.24) \\
1\end{array}$ & $0.027^{*}$ \\
\hline $\begin{array}{l}\text { Medication schedule fit daily } \\
\text { activity } \\
\text { Yes } \\
\text { No }\end{array}$ & $\begin{array}{l}95(39.9) \\
21(77.8)\end{array}$ & $\begin{array}{c}143(60.1) \\
6(22.2)\end{array}$ & $\begin{array}{c}5.27(2.05-13.54) \\
1\end{array}$ & 0.001 & $\begin{array}{c}5.05(1.79-14.21) \\
1\end{array}$ & $0.002^{*}$ \\
\hline $\begin{array}{l}\text { History of } \\
\text { substance abuse } \\
\text { Yes } \\
\text { No }\end{array}$ & $\begin{array}{l}20(83.3) \\
96(39.8)\end{array}$ & $\begin{array}{c}4(16.7) \\
145(60.2)\end{array}$ & $\begin{array}{c}1 \\
7.55(2.50-22.78)\end{array}$ & 0.001 & $\begin{array}{c}1 \\
\mathbf{8 . 0 1}(2.15-29.84)\end{array}$ & $0.002^{*}$ \\
\hline $\begin{array}{l}\text { Concomitantly used medication } \\
\text { Yes } \\
\text { Yes } \quad \text { No }\end{array}$ & $\begin{array}{l}96(47.8) \\
20(31.2)\end{array}$ & $\begin{array}{l}105(52.2) \\
44(68.8)\end{array}$ & $\begin{array}{c}1 \\
2.01(1.12-3.65)\end{array}$ & 0.022 & $\begin{array}{c}1 \\
1.72(0.84-3.51)\end{array}$ & 0.136 \\
\hline $\begin{array}{l}\text { Side effect } \\
\text { Yes } \\
\text { No }\end{array}$ & $\begin{array}{l}79(58.5) \\
37(28.5)\end{array}$ & $\begin{array}{l}56(41.5) \\
93(71.5)\end{array}$ & $\begin{array}{c}1 \\
3.55(2.13-5.92)\end{array}$ & 0.001 & $\begin{array}{c}1 \\
3.89(2.11-7.19)\end{array}$ & $0.001^{*}$ \\
\hline $\begin{array}{l}\text { Patient-health professional rl } \\
\text { ship } \\
\text { Yes } \\
\text { No }\end{array}$ & $\begin{array}{c}93(41) \\
23(60.5)\end{array}$ & $\begin{array}{l}134(59) \\
15(39.5)\end{array}$ & $\begin{array}{c}2.21(1.09-4.46) \\
1\end{array}$ & 0.027 & $\begin{array}{c}0.70(0.25-1.98) \\
1\end{array}$ & 0.506 \\
\hline $\begin{array}{l}\text { Having access to the medical } \\
\text { center } \\
\text { Yes } \\
\text { No }\end{array}$ & $\begin{array}{l}48(33.3) \\
68(56.2)\end{array}$ & $\begin{array}{l}96(66.7) \\
53(36.7)\end{array}$ & $\begin{array}{c}2.57(1.56-4.23) \\
1\end{array}$ & 0.001 & $\begin{array}{c}2.13(1.18-3.86) \\
1\end{array}$ & $0.012^{*}$ \\
\hline $\begin{array}{l}\text { WHO stages } \\
\text { Stage } 1 \\
\text { Stage } 2 \\
\text { Stage } 3 \\
\text { Stage } 4\end{array}$ & $\begin{array}{l}44(47.8) \\
25(29.4) \\
27(51.9) \\
15(51.7)\end{array}$ & $\begin{array}{l}48(52.2) \\
60(70.6) \\
25(48.1) \\
14(48.3)\end{array}$ & $\begin{array}{c}1.17(0.51-2.69) \\
2.57(1.08-6.12) \\
0.99(0.40-2.46) \\
1\end{array}$ & $\begin{array}{l}0.714 \\
0.032 \\
0.986\end{array}$ & $\begin{array}{c}1.21(0.44-3.37) \\
2.23(0.77-6.45) \\
1.14(0.39-3.36) \\
1\end{array}$ & $\begin{array}{l}0.713 \\
0.139 \\
0.807\end{array}$ \\
\hline
\end{tabular}

*statistically significant variables

Table 7: Bivariate and multivariate analysis for factors associated with ART adherence among people living with HIV/AIDS in NEMMGH, Hosanna ( $\mathrm{n}=265$ ), March to April 27, 2015.

Participants who had access to medical center regardless of place and time were 2.13 more likely adhere as compared with those who had no access to medical center $[$ AOR $(95 \% \mathrm{CI})=2.13(1.18-3.86)]$ (Table 7$)$.

\section{Discussion}

Out of 265 patients who undertake ART, 149 patients have adhered to ART in the previous seven days which give overall adherence rate of $56 \%$. This finding was comparable with other studies done in SouthSouth Nigeria 59.9\% [4], Laos 60\% [13], Ghana 62.2\% [14], Cameroon 56.6\% [15], Mekele, Ethiopia 63.4\% [16]. However, adherence level reported in this study was lower than other studies in Ethiopia; Yirgalem Hospital 74.2\% [10], Jimma university specialized hospital 77.9\% [11], Jimma university specialized hospital 75.7\% [17], Addis Abeba 81.2\% [18], Debre Markos [19] and higher than studies done in Namidi Nigeria 14.9\% [20] and Brazil 42\% [21].

The discrepancy might be due to different study designs, different methods of measurement and definitions of adherence used and also by different contexts where patients are. Our study used only selfreports which might overestimate or underestimate the measure of adherence. But multi-method adherence assessment consisting of self- report, the visual analog scale and the pill identification test and pill count was used in other studies. Some use questionnaires (selfreport) prepared by the authors or using standardized questionnaires validated by other, and another have obtained the data through questionnaires in interviews. It is worth noting that some measure the rate of compliance by percentage of drugs taken in the last two, three, four or seven days by patients.

The present study revealed that most of the study participants took prescribed pills as per the instruction while some of them missed it. The main reason for missing doses was forgetting and being busy. This study is in support of a study done in south-south Nigeria [4], Yirgalem hospital [10], Albania [22], Kenya [23] and Addis Ababa [18]. 
Family and social support were likely to increase adherence by odds of 2.07 times [COR $(95 \% \mathrm{CI})=2.07(1.21,3.53)]$. This finding is consistent with other studies $[9,10,12,14,24,25]$. Studies conducted in France [26] and in Cameroon [15] showed lack of support lead to nonadherence and other studies conducted in Ethiopia and other areas witnessed clients with family support were more likely to adhere than those who didn't get family support $[7,11,20,21,27,28]$.

Our study revealed that patients who married were more likely adhered and study done in Albania show that being partnered were associated with poor adherence [22] and were not associated in other studies $[4,14,16,21,24]$. This difference may be due to disclosure of ART use $[29,30]$ and the support they get from their sex partner in our study $[14,24]$.

PLHIV who did face medication side effect had poorer adherence in other studies $[10,14,25,26,29]$. One study documented that no side effect is necessary to facilitate adherence to ART [21]. In agreement with this we found that patients who did not face side effects were 3.89 times more likely adhering [AOR $(95 \% \mathrm{CI})=3.89$ (2.11-7.19)] .In our study, 135 (50.9\%) participants faced side effect from their HAART, which might be contributed a significant amount to medication nonadherence. Among the side effects, fatigue 49 (36.3\%) and headache 22 (16.3\%) took the greater part. This might be due to zidovudine based ARV regimen [11].

A number of studies have shown that active alcohol makes it more difficult for patients to adhere to treatment $[25,26,29]$. In agreement with our study patients who were not alcoholic were 8.01 times more likely to be adhered compared with patients who were alcoholic [AOR $(95 \%$ CI $)=8.01(2.15-29.84]$. This finding is in agreement with studies done in Tapi health center [12] and South Africa [27]. In a study conducted in Botswana, nearly 40 percent of patients admitted had missed a dose because of alcohol consumption [30]. This is due to negative association of adherence with alcohol use which is mostly referred to impaired judgment and forgetfulness.

Participants following medication time schedule were showed statistically significant association with adherence. This variable had a significant association in other reviewed research $[3,9,19]$. Medication schedule fits daily activity had also significant association in this study. Similar finding with the study conducted at Debre Markos hospital [19] but not with a study conducted in Yirgalem hospital [10]. So, health professionals need to draw up the plan that considers the everyday events that occur patients need to understand exactly what the effect of non-adherence is likely to be on their ability to reach those objectives. The patients who found the ART convenient to their daily routine activities stated the advantage to the patient

Disclosure status to anyone was significantly associated with adherence in our study. Studies have shown that HIV disclosure is necessary to facilitate adherence to ART [27] and PLHIV who did not disclose to their family or peers had poorer adherence than those who disclosed their status $[29,30]$ and in other studies, it wasn't associated $[12,14,16,19,24]$. Patients need to be encouraged to disclose their infections so that they will feel less stress going to a center for medication refills. Patients seeking treatment come far from home to increase their level of privacy and avoid the risk of being seen by family members [30]. Our findings suggest that disclosure is an important issue, which should be discussed during the counseling sessions. For those who have not disclosed, providers should explain the importance of disclosure for the success of ART.

Access to health facility regardless of distance and time is of great concern to PLHIV. patients who were willing to take ART they became non-adherent because of difficulties in reaching the treatment centers due to unexpected transport and other strikes; long travel distance; geographical difficulty including lack of transportation services in many remote areas; and the seasonal deterioration of poorer roads during the rainy season. This rationale was confirmed by studies that indicated travel time and access to treatment centers were barriers to ART adherence $[14,26,29]$ and that better access to care was significantly associated with optimal adherence in other studies $[3,11,19]$ and study done in Debre Markos showed no association [19]. In present study participants who had access to medical center regardless of place and time were 2.13 more likely adhere as compared with those who had no access to medical center [AOR $(95 \%$ CI $)=2.13(1.18-3.86)]$. Patients who are from rural areas have difficulties in traveling long distances and finding their travel costs, and have most to gain from nearby ART facilities. Thus, any new policy will need to address this issue and improve access to medical care services by integrating ART treatment into the mainstream of healthcare rather than concentrating treatment in a limited number of ART centers, which may be hard to reach for many patients.

\section{Limitation of the study}

Our study period was short so judging whether a patient was $\geq 95 \%$ adherent or not was a matter of chance and Mediation adherence was assessed through a self-reporting adherence questionnaire in previous week and no other more objective tools such as electronic pill caps, pills counts, and biological methods (patients' viral load and CD4 count) that should be priorities for future studies. This study is a cross-sectional study, which measured adherence at a single time point. However, adherence is a dynamic process that may change over time; thus, it may be that multiple contacts with respondents could have provided more useful information than a single interview.

\section{Conclusion}

The overall medication adherence rate was $56 \%$ which was lower compared with reports from developing countries. Forgetfulness and being busy are the reason for dose non-adherence. The major independently associated factor with ART adherence include no substance abuse, the absence of side-effects, being married, having access to the health center, disclosure status, fitness of daily treatment schedule with daily activity and following specific medication schedule.

In addition, periodic surveillance on adherence rate and predictors of adherence will further increase the adherence rate and also further researches with long study period and with objective tools for assessing adherence should be done to get overall predictors of adherence.

\section{Acknowledgment}

The author would like to express due appreciation for NEMMGH for allowing us to access patients data. We are also grateful to Jimma University for providing us different reference materials and financial support.

\section{Funding}

The study was supported by a research grant from Jimma University (Jimma Ethiopia). The funders had no role in the design of the study and collection, analysis and interpretation of data and in writing the manuscript.

\section{Authors' Contributions}

GM and HJ Conceived and designed the study, analysis, and interpretation of data revised it critically for important intellectual content and approved the final version.

BB: acquired the data, analyzed and interpreted the data, drafted the manuscript and approved the final version.

SE: analyzed the data, interpreted the data, revised it critically for important intellectual content and approved the final version. 
Citation: Billoro BB, Mamo G, Jarso H (2018) Adherence to Antiretroviral Therapy and Associated Factors among HIV Infected Patients in Nigist Eleni Mohammed Memorial General Hospital, Hossana, Southern Ethiopia. J AIDS Clin Res 9: 774. doi: 10.4172/2155-6113.1000774

\section{References}

1. Sow PG, Coume M, Ka O, Gaye A, Vinekar V (2012) Determinants of medication adherence among people living with HIVIAIDS in Senegal. Uni $J$ Edu General Studies 1: 71-76.

2. UNAIDS (2016) Global report: UNAIDS report on global AIDS epidemic 2016 UNAIDS.

3. Adefolalu AO, Nkosi ZZ (2013) The complex nature of adherence in the management of HIVIAIDS as a chronic medical condition. Dis1: 18-35.

4. Oku AO, Owoaje ET, Ige OK, Oyo-Ita A (2013) Prevalence and determinants of adherence to HAART amongst PLHIV in a tertiary health facility in southNigeria. BMC Infect Dis 13: 401

5. Sharma S, Khadga P, Dhungana GP, Chitrakar U (2013) Medication adherence to antiretroviral therapy among patients visiting antiretroviral therapy center at Tribhuvan University Teaching Hospital, Kathmandu Nepal. Kathmandu Univ Med J 11: 50-53.

6. Lee L, Rand CS, Ellen JM, Agwu A (2014) Factors informing HIV providers' decisions to start antiretroviral therapy for young people living with behaviorally acquired HIV. J Adolesc Health 55: 358-365.

7. Tiyou A, Belachew T, Alemseged F, Biadgilign S (2010) Predictors of adherence to antiretroviral therapy among people living with HIVIAIDS in a resourcelimited setting of southwest Ethiopia. AIDS Res Ther 7: 39.

8. Hassan AS, Nabwera HM, Mwaringa SM, Obonyo CA, Sanders EJ, et al. (2014) HIV-1 virologic failure and acquired drug resistance among first-line antiretroviral experienced adults at a rural HIV clinic in coastal Kenya: A crosssectional study. AIDS Res Ther 11: 9.

9. Alagaw A, Godana W, Taha M, Dejene T (2013) Factors associated with antiretroviral treatment adherence among Adult Patients in Wolaita Soddo Hospital. J Trop Dis 1: 125

10. Markos E, Worku A, Davey G (2008) Adherence to ART in PLWHA at Yirgalem Hospital, South Ethiopia. Ethiopian J Health Develop 22: 174-179.

11. Ejigu SH, Rike WA, Angamo MT (2014) Medication adherence and associated factors among patients on highly active antiretroviral therapy in Nekemte Hospital, Ethiopia. Gaziantep Med J 23: 199-208.

12. Tsion D, Serawit D (2014) Rate and predictors of adherence to antiretrovira therapy among clients on antiretroviral therapy at Tepi Health Center, Southwest Ethiopia. Sci Technol Arts Res J 3: 93-98.

13. Hansana V, Sanchaisuriya P, Durham Jo, Sychareun V, Chaleunvong K, et al. (2013) Adherence to antiretroviral therapy (ART) among people living with HIV (PLHIV): A cross-sectional survey to measure in Lao PDR. BMC Public Health 13: 617 .

14. Christian O, Selleh PK, Abledu JK, Fofie CO (2013) Predictors of adherence to antiretroviral therapy among HIVIAIDS patients in the Upper West Region of Ghana. ISRN AIDS : 7.

15. Mbuagbaw L, Thabane L, Ongolo-Zogo P, Yondo D, Noorduyn S, et al. (2012) Trends and determining factors associated with adherence to antiretroviral therapy (ART) in Cameroon: A systematic review and analysis of the CAMPS trial. AIDS Res Ther 9: 37.
16. Hailasillassie K, Etana B, Alemayehu M, Fisseha G (2014) Factors associated with adherence to highly active antiretroviral therapy among adult HIVIAIDS patients in Mekelle Hospital Northern Ethiopia. Sci J Public Health 2: 367-372

17. Amberbir A, Woldemichael K, Getachew S, Girma B, Deribe K (2008) Predictors of adherence to antiretroviral therapy among HIV infected persons: A prospective study in Southwest Ethiopia. BMC Public Health 8: 265

18. Tadious Y, Davey G (2006) Antiretroviral treatment adherence and its correlates in Addis Ababa, Ethiopia. Ethiopian Med J 44: 237-244.

19. Asmare M, Aychiluhem M, Ayana M, Jara D (2014) Level of ART adherence and associated factors among HIV seropositive adult on highly active antiretroviral therapy in Debre Markos Referral Hospital, Northwest Ethiopia. J Antivir Antiretrovir 6: 120-126.

20. ljeoma O, Uchenna O, Anthonia C, Peace I (2013) Nonadherence factors and sociodemographic characteristics of HIV-infected adults receiving antiretroviral therapy in Nnamdi Azikiwe University Teaching Hospital, Nnewi, Nigeria. ISRN AIDS: 8

21. Casotti $A$ (2011) Factors associated with adherence to HAART in patients with HIVIAIDS, Brazil. DST-J bras Doenças Sex Transm 23: 215-221.

22. Morrison SD, Rashidi V, Sarnquist C, Banushi VH, Hole MK, et al. (2014) Antiretroviral therapy adherence and predictors to adherence in Albania: A cross-sectional study. J Infect Dev Ctries 8: 853-862.

23. Wakibi SN, Zipporah WN, Mbugua GG (2011) Factors associated with nonadherence to highly active antiretroviral therapy in Nairobi, Kenya. AIDS Res Ther 8: 43.

24. Mitiku H, Abdosh T, Teklemariam Z (2013) Factors affecting adherence to antiretroviral treatment in Harari National Regional State, Eastern Ethiopia. ISRN AIDS: 7.

25. Wasti SP, Simkhada P, Randall J, Freeman JV, van Teijlingen E (2012) Factors influencing adherence to antiretroviral treatment in Nepal: A Mixed-Methods Study. PLoS ONE 7: e35547.

26. Protopopescu C, Raffi F, Roux P, Reynes J, Dellamonica P, et al. (2009) Factors associated with non-adherence to long-term highly active antiretroviral therapy: A 10 year follow-up analysis with correction for the bias induced by missing data. J Antimicrob Chemother 64: 599-606.

27. Ross AJ, Aung M, Campbell L, Ogunbanjo GA (2011) Factors that positively influence adherence to antiretroviral therapy by HIV and/or AIDS patients and their caregivers. Afr J Prm Health Care Fam Med 3: 196.

28. Rougemont M, Stoll BE, Elia N, Ngang P (2009) Antiretroviral treatment adherence and its determinants in Sub-Saharan Africa: A prospective study at Yaounde Central Hospital, Cameroon. AIDS Res Ther 6: 21.

29. Mills EJ, Nachega JB, Bangsberg DR, Singh S, Rachlis B, et al. (2006) Adherence to HAART: A systematic review of developed and developing nation patient-reported barriers and facilitators. PLoS Med 3: e438.

30. Reda AA, Biadgilign S (2012) Determinants of adherence to antiretrovira therapy among HIV-Infected patients in Africa. AIDS Res Treat: 8. 